\title{
Acoustic Trauma
}

National Cancer Institute

\section{Source}

National Cancer Institute. Acoustic Trauma. NCI Thesaurus. Code C50453.

Injury to the hearing mechanisms within the inner ear, caused by excessively loud noise. 